\title{
Removal of Silicon Oil from the Vitreous Cavity via the Anterior Chamber. Eight Years of the Author's Experiences
}

\author{
Adam Cywinski
}

\section{ABSTRACT}

Purpose: To evaluate usefulness and safety of removal of silicone oil from the vitreous cavity via the anterior chamber.

\begin{abstract}
Methods: The procedures were performed between 2012 and 2020 in 31 patients who required endotamponade with the use of light silicone oils. In most of the patients, the procedure was combined with cataract removal, secondary lens implantation or transscleral fixation of an artificial lens. A passive bimanual technique was used to replace silicone oil with fluid.
\end{abstract}

Results: The procedure to remove silicon oil via the anterior chamber proceeded without serious intra- and postoperative complications. There were no hypotony observed in first postoperative day.

Conclusion: Patients eligible for the procedure are those who have preoperative indications for silicone oil removal and whose removal path via the anterior chamber is not associated with a special need to remove the lens from the eye.

Keywords: silicon oil removal, posterior vitrectomy, cataract, secondary lens implantation, hypotony, aphakia.

\section{INTRODUCTION}

Removal of silicone oil from the vitreous cavity constitutes a permanent part of retinal surgery. Silicone oil endotamponade is used in many diseases, the most frequently mentioned ones being retinal detachment, ocular complications of diabetes or eye trauma [1]. Due to their gravity, silicone oils can be divided into light and heavy silicone oils. Heavy silicon oil is a combination of oil 5000 with perfluorohexylacetate (e.g., Densiron) and is characterized by increased specific gravity and viscosity [2], [3].

The properties of silicone oil determine the method of its removal. Light silicone oil can be removed in many ways. The most common is pars plana, active aspiration using the 2 or 3 port technique. Another option is passive oil removal in the same way. However, this is very time consuming. The size of the removal path (25G-20G), oil viscosity as well as its volume are the main factors determining the speed of removal from the eye. In the case of heavy silicone oil, due to its high specific gravity, greater than specific gravity of water, the technique of active aspiration from the eye constitutes the only possible way of its removal [4], [5].

There is another method of light silicone oil removal - via the anterior chamber. This method is rarely used, which is confirmed by a small number of publications [6]. Regarding postoperative complications, one of the most frequently observed is the presence of residual oil droplets in the vitreous cavity. Even multiple air-fluid exchanges cannot ensure that silicone oil particles will not appear in the days following treatment. Another complication is transient hypotony. More serious complications include recurrent retinal detachment or subsequent glaucoma [1]-[7]. In the case of silicone oil removal via the anterior chamber, $\mathrm{Xu}$ et al. noted a higher percentage of recurrent retinal detachment after oil removal via the anterior chamber compared to the conventional method. In the study group, the authors used the technique of active oil aspiration from the eye.

\section{OBJECTIVE}

Retrospective evaluation of the effectiveness and safety of the procedure of passive silicone oil removal from the vitreous cavity via the anterior chamber followed by corneal incision. Most of the procedures were combined procedures, associated with the implantation of an artificial lens into the eye.

\section{MATERIAL AND METHOD}

The procedures were performed by one surgeon (Dr. Cywinski) between 2012 and 2020 in a private medical centre, Silesian Eye Treatment Centre, Zory, Poland. Silicon oil (SO) removal in this modification was performed in 31 
patients ( 31 eyes). The age of the patients ranged between 38-67 years. Most of posterior vitrectomy procedures (26 eyes), including those combined with cataract removal, were performed by the same surgeon, which means that the plan and method of treatment were standardized. The cause of posterior vitrectomy surgery was retinal detachment diagnosed in 22 patients (eyes) and ocular complications of diabetes -9 eyes. The SO was removed within 2 to 15 months of administration, on average after 3 months. The first procedure to remove SO via the anterior chamber was performed in an aphakic eye (complete absence of the posterior capsular bag) after an eye trauma, in which transscleral fixation of an artificial lens was planned and then performed. So, with the viscosity of 1300 centistokes (cSt) was removed via a $2.5 \mathrm{~mm}$ corneal incision. All procedures, except two (SO removal in high myopia), were combined with other procedures. The type of combined procedures performed, the number of eyes treated, and the type of oil removed are listed in Table I.

TABLE I: INFORMATION INCLUDING TYPE OF SURGERY, NUMBER OF EYES AND KIND OF SILICON OIL, WHICH WAS REMOVED FROM THE EYE

\begin{tabular}{ccc}
\hline \hline Type of surgery & No of eyes & $\begin{array}{c}\text { Type of silicon oil } \\
(\mathrm{cSt})\end{array}$ \\
\hline $\begin{array}{c}\text { Oil removal alone } \\
\text { Oil removal combined } \\
\text { with transscleral fixation } \\
\text { of artificial lens }\end{array}$ & 2 & $2 \times 1300$ \\
$\begin{array}{c}\text { Oil removal in aphakic } \\
\text { eye combined with } \\
\text { posterior capsulorhexis } \\
\text { and secondary lens } \\
\text { implantation }\end{array}$ & 4 & $3 \times 1300$ \\
$\begin{array}{c}\text { Oil removal combined } \\
\text { with cataract removal, } \\
\text { posterior capsulorhexis } \\
\text { and lens implantation }\end{array}$ & 15 & $4 \times 1300$ \\
\hline \hline
\end{tabular}

Removal of crystalline lens or progressive cataract without subsequent implantation of an artificial lens, accompanying posterior vitrectomy resulted from the inability to precisely calculate the artificial lens power because of the retinal detachment involving the macula. In most cases, after the lens was removed, a capsular tension ring was inserted. The artificial lens power was calculated when the retina was completely attached including macular area.

Qualification for surgery includes several permanent steps. In addition to routine activities including the examination of visual acuity to near and far distances with and without correction, examination of the anterior and posterior segment of the eye after pupil dilatation, special attention is paid to:

○ Measurement of intraocular pressure (IOP).

$\circ$ An examination of the eye condition for the presence of silicone oil emulsification.

- An examination of the retina - it has to be attached. In the presence of cataract, the lens should be transparent enough not to impair proper examination of the fundus details.

- Assessment of the number of endothelial cells of the cornea. Due to the low number of endothelial cells, below $1000 / \mathrm{mm}^{2}$, oil removal using this method was refrained from.
Standard treatment procedures included:

1) Local anaesthesia.

2) Three corneal incisions - the main one, $2.0-2.5 \mathrm{~mm}$, to insert the artificial lens and remove $\mathrm{SO}$, and two lateral incisions.

3) Posterior capsulorhexis in the eyes with the preserved posterior capsule.

4) Passive removal of silicone oil using active irrigation, introducing fluid into the vitreous cavity via posterior capsulorhexis. Ringer's solution replaces oil in the passive exchange process.

5) Bimanual technique - including irrigation in combination with the pushing of a spatula on the lower edge of the main corneal incision in order to facilitate the drainage of oil from the eye.

6) Secondary implantation of a lens into the capsule or on the anterior capsule, or transscleral fixation of an artificial lens. Currently, Carlevale by Soleko [8] is the preferred model for intrascleral fixation.

7) The previous step is usually preceded by introducing a maintainer into the anterior chamber in order to save good eye tension.

8) Hydration of corneal wounds without using corneal stitches.

\section{RESULTS}

The anatomical condition of the eye, IOP values achieved on the first postoperative day and 30 days after surgery like also observed complications were analysed.

The procedure to remove SO via the anterior chamber proceeded without serious intra- and postoperative complications.

One of the most important observations worth paying attention to is the lack of hypotony on the first day after surgery. In no case pressure lower than $10 \mathrm{mmHg}$ was observed, and in 8 cases pressure was above $22 \mathrm{mmHg}$.

\section{A. Intraoperative Complications}

Partial irises prolapse via the main corneal incision during oil removal was observed in 4 cases (12\%). This condition led to a local loss of pigment epithelium od the iris. Changing the technique (using a wider spatula) allowed for the avoidance of this complication during subsequent treatments.

\section{B. Postoperative complications}

1) Transient corneal oedema, 25 eyes $(80.6 \%)$. Oedema was most pronounced in the eyes in which transscleral fixation and cataract extraction was combined with SO removal. Oedema has disappeared for 5-7 days. Administration of $5 \% \mathrm{NaCl} 3-4$ times per day helps to resolve this problem

2) Bleeding into the anterior chamber and vitreous observed in 3 eyes $(9.6 \%)$ that underwent transscleral fixation (1) and intrascleral fixation (2), which subsided within 5-7 days after the procedure.

3 ) Increase of IOP to the value of $24-34 \mathrm{mmHg}-7$ eyes $(22.5 \%)$. Due to the significantly increased emulsification process, already observed before the procedure, 4 patients $(12.9 \%)$ required continuous use of topical antiglaucomatous eyedrops. In one patient with diabetic retinopathy, due to the severe increasing of IOP values (about $40 \mathrm{mmHg}$ ), which did 
not decrease after the use of topical and systemic antiglaucomatous agents, Ahmed valve implantation was performed, in the author's own modification (the valve model intended for the anterior chamber is inserted into the vitreous cavity) [9].

An example of treatment involving retinal attachment followed by SO removal via the anterior chamber in combination with secondary implantation of a bifocal lens is shown below.

https://www.youtube.com/watch? $\mathrm{v}=\mathrm{WkOO}$ RvSm8k\&t=1s

In this patient, one month after surgery, visual acuity to far distances reached without correction, a value up to 0.3 in $\log$ Mar, and to near distances a value of D-0.5 in Snellen. The patient was a little bit myopic after secondary lens implantation.

The follow-up period was from 2 months to 7 years. During this period, no patients experienced recurrent retinal detachment. Four patients (12.9\%) with ocular complications of diabetes experienced multiple recurrent intravitreal haemorrhages within 14 months of SO removal. The haemorrhages resolved spontaneously.

\section{DISCUSSION}

Is it worth using the technique of removing silicone oil via the anterior chamber? Taking into account my own experience, I can say that it is a safe and effective technique. What are the main benefits of this procedure?

$\circ$ No need for periocular anaesthesia required in the case of posterior vitrectomy performed under local anaesthesia.

○ No need to use instruments necessary to remove oil through the pars plana.

- Reduction of the risk associated with the formation of iatrogenic tears in the retina during the insertion of the trocars through the pars plana.

- Lack of postoperative hypotony. Similar results were achieved by Zhang et al. who examined the values of intraocular pressure in a group subjected to oil removal via a $3.5 \mathrm{~mm}$ corneal incision in relation to oil removal by $20 \mathrm{G}$ pars plana surgery. The authors point to choroidal detachment as the main cause of hypotony [10]. Kim SW et al. indicate that hypotony is more often observed in eyes with high myopia [11]. In two patients included in this study, oil removal via a corneal incision did not result in hypotony. Additionally, according to the author of this article, an additional cause is leakage of postoperative scleral wounds in the eyes in which the sutureless technique was used. This condition is more often observed in young people, in whom the sclera is very elastic, so the edges of the scleral wound are less adherent, and in eyes in which the 25-23G sutureless technique was used. Paradoxically, hypotony is less common with the $20 \mathrm{G}$ technique due to the more "comma-like" shape and better adhesion of the edges of the scleral wounds. Similar observations were reported by Byeon et al. [12].

- Significant reduction of the procedure duration. So, removal via a $2-2.5 \mathrm{~mm}$ incision is much more effective than via a pars plana 0.9-0.5 mm incision, even when using the active aspiration technique [6].
In the case of combined procedures, eye traumatization is much smaller - fewer incisions of the eyeball are required.

- Smaller amount of silicone SO droplets visible in the vitreous cavity in the following days after surgery. This may be due to the accumulation of oil droplets in the area under the lens capsule that is above the entries made through the pars plana. Additionally, such a method of oil removal allows for more effective removal of oil droplets, present in the anterior chamber.

o No recurrent retinal detachment. It is difficult to unequivocally comment on more than $15 \%$ of recurrent retinal detachment, described by $\mathrm{Xu}$ et al. after oil removal via the anterior chamber [6]. Such a large difference, i.e., the lack of recurrent retinal detachment in the described group, may be due to the fact that all procedures were performed by one surgeon, including qualification for silicone oil removal, or the use of passive oil removal technique, not active aspiration as described by $\mathrm{Xu}$.

The oil removal procedure via the anterior chamber can be considered an extended form of lens surgery with all its benefits rather than pars plana surgery.

The main "disadvantage" associated with this method of oil removal from the eye is the inability to assess the condition of the retina to the extent allowing for the intraoperative introduction of endoillumination into the vitreous cavity. However, a detailed fundus oculi examination, which should be performed in every patient qualified for oil removal, should be sufficient to make this decision.

\section{CONCLUSIONS}

Removal of silicone oil from the vitreous cavity via the anterior chamber seems to be an effective, efficient, and safe method. It allows not only to reduce eye traumatization, but also to shorten the time needed to perform this procedure. Additional benefits are economic in nature, which should be important when planning and choosing the best treatment method. The lack of postoperative hypotony in the first days after surgery additionally favours this procedure as a safe method of removing silicone oil from the eye.

\section{REFERENCES}

[1] Barca F, Caporossi T, Rizzo S. Silicone oil: different physical proprieties and clinical applications. Biomed Res Int.

[2] Prazeres J, Magalhães O Jr, Lucatto LF, Navarro RM, Moraes NS, Farah ME, Maia A, Maia M. Heavy silicone oil as a long-term endotamponade agent for complicated retinal detachments. Biomed Res Int.

[3] Caporossi T, Franco F, Finocchio L, et al. Densiron 68 heavy silicone oil in the management of inferior retinal detachment recurrence: analysis on functional and anatomical outcomes and complications. Int J Ophthalmol. 2019;12(4):615-620.

[4] Kaya M, Özyurt A, Öztürk AT, Er D, Kaynak S, Koçak N. Active Silicone Oil Removal with a Transconjunctival Sutureless System: Is the 23-Gauge System Safe and Effective? Turk J Ophthalmol. 2016;46(1):11-15

[5] Siyal NA, Hargun LD, Wahab S. Passive removal of silicone oil through 23gauge transconjunctival sutureless vitrectomy system. Pak J Med Sci. 2016;32(3):652-656. 
[6] Xu W, Cheng W, Zhuang H, Guo J, Xu G. Safety and efficacy of transpupillary silicone oil removal in combination with micro-incision phacoemulsification cataract surgery: comparison with 23-gauge approach. BMC Ophthalmol. 2018 Aug 15;18(1):200.

[7] Miller JB, Papakostas TD, Vavvas DG. Complications of emulsified silicone oil after retinal detachment repair. Semin Ophthalmol. 2014 Sep-Nov;29(5-6):312-8.

[8] Cywinski A. Sutureless intrascleral fixation of the Carlevale (Soleko) intraocular lens in the correction of aphakia. Own experience. Cywinski A. European Journal of Medical and Health Sciences, 2(4).

[9] Cywinski A, Bednarski L. A Treatment of Refractory Glaucoma in Pseudophakic Patients after Posterior Vitrectomy using the Ahmed Valve in an Own Modification. International Journal of Research Studies in Medical and Health Sciences. Volume 4, Issue 6, 2019.

[10] Zhang X, Chen B, Yang H, Song Y, Zhang D, Soetikno BT, Sun X. The Correlation of Pars Plana Incision and Transient Hypotony After Silicone Oil Removal. Ophthalmic Surg Lasers Imaging Retina. 2018 Sep 1;49(9) e44-e51.

[11] Kim SW, Oh J, Yang KS, Kim MJ, Rhim JW, Huh K. Risk factors for the development of transient hypotony after silicone oil removal. Retina. 2010 Sep;30(8):1228-36.

[12] Byeon SH, Lew YJ, Kim M, Kwon OW. Wound leakage and hypotony after 25-gauge sutureless vitrectomy: factors affecting postoperative intraocular pressure. Ophthalmic Surg Lasers Imaging. 2008 MarApr;39(2):94.

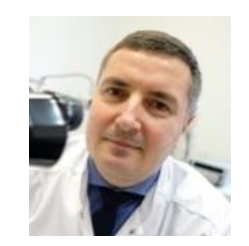

Adam Cywinski, date of birth 22.09.1967. Medical Director and founder of private medical center, Silesian Eye Treatment Centre, Zory, Poland. Consultant of posterior segment diseases. He graduated Silesian Medical Academy in Katowice and gained medical degree in the biggest Clinic of Ophthalmology, also in Katowice (Poland). He doctorated in surgical topic "Posterior vitrectomy with gas endotamponade and ILM peeling in IV stage macular hole, in 2003. As a surgeon he made a few thousands posterior vitrectomy procedures, including macular diseases, retinal detachment, diabetic and posttraumatic cases. His next interest is anterior segment surgery with special focus on reconstruction of the iris and lens surgery with using of premium class lenses. Dr Cywinski is a member of PTO, AAO, ESCRS, Euretina. 